\title{
Efficient and accurate determination of genome-wide DNA methylation patterns in Arabidopsis thaliana with enzymatic methyl sequencing
}

\author{
Suhua Feng ${ }^{1,2 \dagger}$, Zhenhui Zhong ${ }^{1 \dagger}$, Ming Wang ${ }^{1}$ and Steven E. Jacobsen ${ }^{1,2,3^{*}}$ (D)
}

\begin{abstract}
Background: 5' methylation of cytosines in DNA molecules is an important epigenetic mark in eukaryotes. Bisulfite sequencing is the gold standard of DNA methylation detection, and whole-genome bisulfite sequencing (WGBS) has been widely used to detect methylation at single-nucleotide resolution on a genome-wide scale. However, sodium bisulfite is known to severely degrade DNA, which, in combination with biases introduced during PCR amplification, leads to unbalanced base representation in the final sequencing libraries. Enzymatic conversion of unmethylated cytosines to uracils can achieve the same end product for sequencing as does bisulfite treatment and does not affect the integrity of the DNA; enzymatic methylation sequencing may, thus, provide advantages over bisulfite sequencing.

Results: Using an enzymatic methyl-seq (EM-seq) technique to selectively deaminate unmethylated cytosines to uracils, we generated and sequenced libraries based on different amounts of Arabidopsis input DNA and different numbers of PCR cycles, and compared these data to results from traditional whole-genome bisulfite sequencing. We found that EM-seq libraries were more consistent between replicates and had higher mapping and lower duplication rates, lower background noise, higher average coverage, and higher coverage of total cytosines. Differential methylation region (DMR) analysis showed that WGBS tended to over-estimate methylation levels especially in $\mathrm{CHG}$ and $\mathrm{CHH}$ contexts, whereas EM-seq detected higher CG methylation levels in certain highly methylated areas. These phenomena can be mostly explained by a correlation of WGBS methylation estimation with GC content and methylated cytosine density. We used EM-seq to compare methylation between leaves and flowers, and found that CHG methylation level is greatly elevated in flowers, especially in pericentromeric regions.
\end{abstract}

Conclusion: We suggest that EM-seq is a more accurate and reliable approach than WGBS to detect methylation. Compared to WGBS, the results of EM-seq are less affected by differences in library preparation conditions or by the skewed base composition in the converted DNA. It may therefore be more desirable to use EM-seq in methylation studies.

Keywords: DNA methylation, Bisulfite sequencing, WGBS, EM-seq, TET, APOBEC, Arabidopsis flowers, Arabidopsis leaves

\footnotetext{
*Correspondence: jacobsen@ucla.edu

†Suhua Feng and Zhenhui Zhong contributed equally to this work

1 Department of Molecular, Cell and Developmental Biology, University of California at Los Angeles, Los Angeles, CA 90095, USA

Full list of author information is available at the end of the article
}

\section{Introduction}

The fifth carbon position of cytosine in DNA can be covalently modified by the addition of a methyl group to form 5-methylcytosine (5-mC). DNA methylation that takes place at cytosine residues which are followed by guanine 
is termed CG methylation and is conserved in most eukaryotes. Non-CG methylation, where modification occurs in $\mathrm{CHG}$ and $\mathrm{CHH}$ contexts (where $\mathrm{H}$ corresponds to $\mathrm{A}$, $\mathrm{T}$, or $\mathrm{C}$ residues), occurs in plants and many other organisms. $\mathrm{CHH}$ methylation is also called asymmetrical methylation. DNA methylation is typically associated with the silencing of genes and repetitive DNAs such as transposable elements; however, expressed genes are also found to be methylated. DNA methylation is involved in a large number of cellular processes including genomic imprinting, $\mathrm{X}$ chromosome inactivation, embryonic development, and transcriptional regulation of developmentally important genes, as well as in ensuring genome integrity and protecting against invasive DNAs [1-5].

The first step in the study of DNA methylation is to determine whether or not a given cytosine residue is methylated. Indirect approaches to measure methylation include pull-down assays with methylation-specific antibodies and methyl-binding proteins, and restriction digestion with enzymes with preferences for or against methylcytosines [6]. The direct approach, which can achieve single-nucleotide resolution, is sequencing. Since methylated cytosine pairs with guanine in the same way unmethylated cytosine does, traditional sequencing methods (based on base-pairing) are not able to differentiate between methylated and unmethylated cytosines. To solve this problem, sodium bisulfite can be used to convert unmethylated cytosines to uracils, which are amplified as thymines in PCR; because methylated cytosines do not react with sodium bisulfite, they remain as cytosines in the sequence. Thus, thymines detected in bisulfite sequencing correspond to either thymines or unmethylated cytosines in the original DNA, and alignment with the original template sequence easily differentiates between them [7]. Since its development a little more than a decade ago, whole-genome bisulfite sequencing (WGBS) has been successfully used to survey DNA methylation on a genome-wide scale [8-11]. While WGBS (which combines bisulfite treatment with high-throughput sequencing) is the gold standard for measuring genome-wide methylation, it has several shortcomings. After bisulfite conversion, DNA becomes C-poor, which can result in difficulties for polymerase reactions, as well as with sequencing machines, basecallers, and aligners. Although recent improvements in PCR reagents, sequencing hardware/software, and bioinformatics tools have helped to alleviate these difficulties, two fundamental problems remain. First, bisulfite degrades the majority of the DNA during the conversion process (due to backbone scission induced by depyrimidination and perhaps depurination as well); second, bisulfite preferentially damages DNA at unmethylated cytosines via depyrimidination (more effectively than at methylated cytosines) [12, 13]. These properties of bisulfite treatment make it challenging to perform WGBS from tissues that have limited starting material, and create a bias that can result in an over-estimation of methylation level by WGBS.

In addition to chemicals like bisulfite, DNA bases can also react with enzymes. For example, 5 - $\mathrm{mC}$ can be converted to 5-hydroxymethylcytosine $(5-\mathrm{hmC})$, then to 5 -formylcytosine (5-fC), and finally to 5-carboxylcytosine $(5-\mathrm{caC})$ in a cascade of reactions regulated by the ten-eleven translocation (TET) family of dioxygenases $[14,15]$. Both methylated and unmethylated cytosines can be deaminated by the apolipoprotein $\mathrm{B}$ mRNA editing enzyme catalytic polypeptide-like 3A (APOBEC3A) to generate thymines and uracils, respectively [16-18]. Interestingly, APOBEC3A has only negligible cytidine deaminase activity toward the TET-oxidized methylcytosines, 5-hmC, 5-fC, and 5-caC [19]. This creates an opportunity to use a combination of TET and APOBEC3A to differentiate between methylated and unmethylated cytosines.

Recently, an enzymatic methyl-seq (EM-seq) technique was developed, which uses TET2 in the first enzymatic step to oxidize methylated cytosines and APOBEC2 in the second enzymatic step to convert unmethylated cytosines to uracils [20]. During the subsequent PCR amplification, oxidized methylcytosines form base pairs with guanines and uracils form base pairs with adenines. Since the end products of WGBS and EMseq are the same (methylated cytosines stay as cytosines and unmethylated cytosines appear as thymines in the sequence), the same analysis tools can be used. Because enzymatic reactions are non-destructive, EM-seq promises better yield and higher accuracy in the measurement of methylation levels [20]. In this study, we employed EM-seq to study DNA methylation in Arabidopsis thaliana and compared EM-seq results to those from WGBS. We also used EM-seq to examine the methylation differences between flowers and leaves in Arabidopsis.

\section{Results}

\section{Generating and sequencing EM-seq and WGBS libraries}

To systematically compare EM-seq and WGBS, we prepared Illumina sequencing libraries from different amounts of genomic DNA extracted from Arabidopsis flowers-25 ng, $50 \mathrm{ng}, 150 \mathrm{ng}$, or $400 \mathrm{ng}$ (to encompass the range of starting material amounts that are typically used in an Arabidopsis methylome study). This was followed by either enzymatic (TET2 followed by APOBEC3A) or bisulfite treatment, and PCR amplification of the converted products (for 6,12 , or 18 cycles) to complete the library preparations (Additional file 1: Fig. S1, see also Methods). Two replicates were performed 
for each condition. EM-seq libraries consistently showed higher mapping rates and lower duplication rates than WGBS libraries, and the variation between EM-seq libraries was smaller than the variation between WGBS libraries (Fig. 1ab, Additional file 2: Table S1a). In addition, within WGBS libraries, higher mapping rates and lower duplication rates (manifested by higher effective read rate) were generally associated with moderate input amounts and lower PCR cycle numbers (Additional file 2: Table S1a). One of the causes of false methylation reporting in bisulfite sequencing is non-conversion, in which the two strands of DNA occasionally fail to fully denature during bisulfite treatment and are thus resistant to bisulfite conversion [21]; this leads to the detection of several adjacent un-converted cytosines. In Arabidopsis, we previously introduced a filter that discards sequencing reads with three or more consecutive methylated cytosines in the $\mathrm{CHH}$ context [8]. This non-conversion filter works well in Arabidopsis, since $\mathrm{CHHs}$ are rarely methylated above $10 \%[22,23]$, so the chance of observing three consecutive methylated $\mathrm{CHHs}$ is below $0.1 \%$ and only a small number of real methylation signatures are discarded. However, it is not practical to use this filter in organisms that have high levels of $\mathrm{CHH}$ methylation. Very few EM-seq reads (1.56\%-2.01\%) eligible for removal by the non-conversion filter were identified, while the filtered rates for WGBS libraries were much higher and showed greater variation (2.62\% to $13.41 \%)$ (Fig. 1c, Additional file 2: Table S1b). This suggests that the EM-seq method is generally free of the notorious non-conversion problem that is frequently observed in WGBS, and is, therefore, useful for methylation detection in organisms in which use of an arbitrary non-conversion filter is not suitable. Consistent with this, we found that virtually no methylated cytosines were detected from the unmethylated Arabidopsis chloroplast genomes in any EM-seq library, a result that is only achieved or approached by the best WGBS library (400 ng input with 12 cycles of PCR) (Additional file 1: Fig. S2, Additional file 2: Table S1c). This indicates that EM-seq has much lower background noise levels than WGBS.

As expected from these comparisons, EM-seq also displays advantages in terms of genomic coverage (Fig. 1d). Perhaps more importantly, EM-seq is able to cover $22.07 \%, 22.10 \%$, and $23.47 \%$ more CG, CHG, and $\mathrm{CHH}$ sites, respectively, than WGBS (Fig. 2a); these numbers are consistent with the previous findings of EM-seq in human cells [20] and the manufacturer's description of the product [24]. All EM-seq libraries exhibit similar cytosine coverage (CG: ranging from 5,556,957 to 5,602,669, CHG: ranging from 6,090,541 to 6,128,646, $\mathrm{CHH}$ : ranging from $31,123,001$ to $31,315,262)$, while different preparation conditions clearly affect the performance of WGBS libraries (CG: ranging from 3,922,759 to $5,165,506$, CHG: ranging from $4,26,9441$ to $5,664,610$, CHH: ranging from 22,080,267 to 28,678,168) (Fig. 2a).

Next, we examined the dependence of coverage on nucleotide composition. Dinucleotide profiles suggest that EM-seq has even coverage and is minimally affected by different dinucleotide combinations. In contrast, WGBS libraries show enrichment for dinucleotides containing Gs and depletion for dinucleotides contains Cs (Fig. 2b), consistent with the damaging effect of sodium bisulfite on unmethylated cytosines [13]. These biases are more pronounced in libraries with 18 cycles of PCR (Fig. 2b), indicating that PCR amplification during WGBS library preparation favors unconverted DNA over converted DNA, due to the low melting temperature
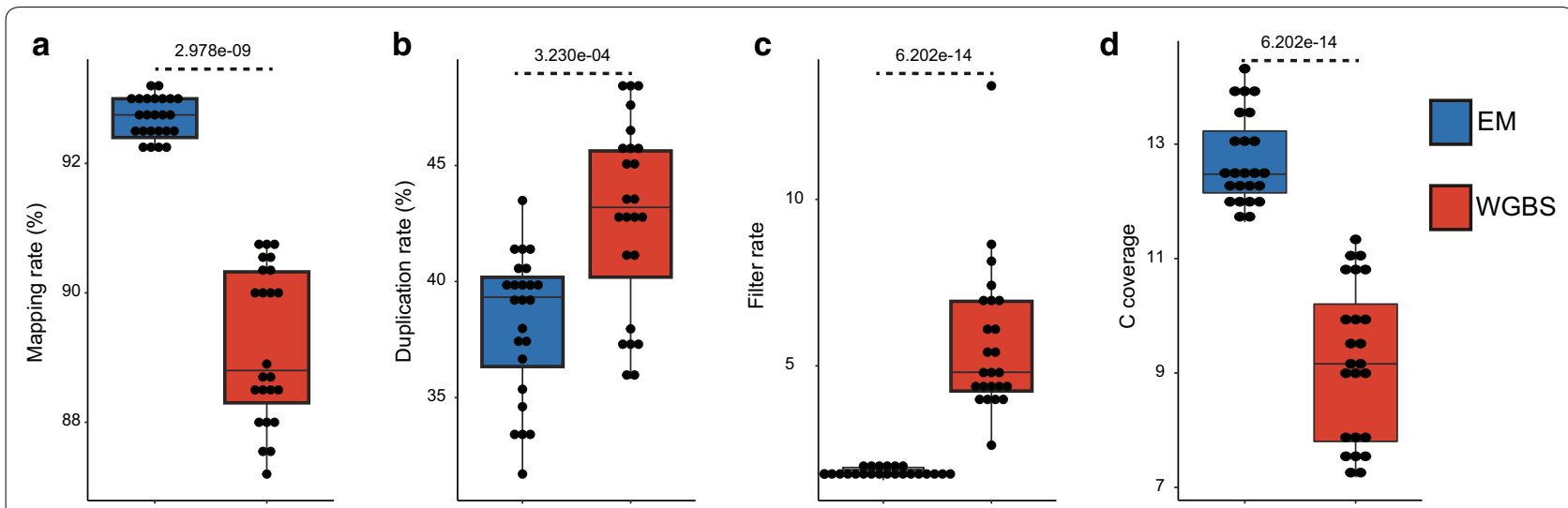

Fig. 1 Quality comparison between EM-seq and WGBS. Boxplots showing: a mapping rates of EM-seq and WGBS. $\mathbf{b}$ duplication rates of EM-seq and WGBS. c read filtering rates of EM-seq and WGBS. Reads with three or more consecutive methylated CHH sites are considered as non-converted reads and removed from subsequent analyses. $\mathbf{d}$ Boxplot showing average coverages of methylated cytosines in EM-seq and WGBS. In a-d, blue box represents EM-seq and red box represents WGBS. The $P$ values indicated at the top of each plot were estimated using a Student's $t$-test 


\section{(See figure on next page.)}

Fig. 2 Coverage comparison between EM-seq and WGBS. a Boxplots showing numbers of methylated CG, CHG, and CHH cytosines identified by EM-seq and WGBS. Blue box represents EM-seq and red box represents WGBS. The $P$ values shown at the top were estimated using a Student's t-test. $\mathbf{b}$ Average coverage levels of different dinucleotides in EM-seq and WGBS. c Average coverages of genome-wide 400 bp bins, ranked by GC content, in EM-seq and WGBS. In $\mathbf{b}$ and c, coverage levels are normalized with expected coverages (total reads over reference genome). Colored lines represent different library preparations

and thermostability of AT pairs $[25,26]$. This can further negatively affect accurate estimation of methylation level (see below). Similar biases were not observed in EM-seq libraries, although the product of TET2/APOBEC3A conversion is the same as that of bisulfite conversion, which suggests that the polymerase used to amplify EMseq libraries is superior to the one used for WGBS, in terms of avoiding the introduction of base biases. As previously shown for a library preparation kit similar to the one used in our study [27], WGBS libraries show enrichment for dinucleotides containing only A or T (Fig. 2b). We then decided to look directly at the dependence of coverage on GC content. Again, EM-seq libraries show more even profiles over the majority of the GC content range than do WGBS libraries (Fig. 2c). WGBS libraries have clearly higher coverage in AT-rich regions than in GC-rich regions (Fig. 2c), a known issue for bisulfite sequencing $[20,27]$ that is discussed above (Fig. 2b).

Overall, the quality metrics of our EM-seq libraries encouraged us to further explore whether it measures methylation more accurately than WGBS in Arabidopsis.

\section{Arabidopsis DNA methylation levels measured by EM-seq and WGBS}

We compared levels of CG, CHG, and CHH methylation measured by EM-seq and by WGBS and noted that EM-seq-measured DNA methylation levels are lower (Fig. 3a-c, Additional file 3: Table S2), even if background noise is considered (see Additional file 1: Fig. S2). This is consistent with the previous results obtained by the manufacturer for Arabidopsis [24]. Total DNA methylation levels estimated from EM-seq data (Fig. 3d) are close to the levels previously detected by liquid chromatography-mass spectrometry (LC-MS) in Arabidopsis [24]. We observed that increasing the number of PCR cycles led to higher methylation levels in the respective WGBS libraries (especially for libraries with 18 cycles of PCR; Fig. 3a-d, Additional file 3: Table S2) and reasoned that this likely reflects the above-mentioned preference of PCR amplification during WGBS library preparation for unconverted DNA (see Fig. 2b). To further test this hypothesis, we analyzed the correlation of methylation level with density of methylated cytosines in both EM-seq and WGBS. For this analysis, we picked the best WGBS library (400 ng input with 12 cycles of PCR) (see Additional file 1: Fig. S2, Additional file 2: Table S1c) and its EM-seq counterpart. As Fig. 3ef reveals, for CHG and $\mathrm{CHH}$ methylations, the differences in methylation levels between EM-seq and WGBS increases with cytosine methylation density, which is the expected result based on our hypothesis. Much less difference between EM-seq and WGBS was observed for CG methylation, possibly because CG methylation is more or less bimodal (either completely unmethylated or completely methylated) [8], and thus, the PCR bias in WGBS library preparation toward methylated CG sites would have less influence on CG methylation percentages. Nonetheless, CG methylation levels still appear to be moderately over-estimated by WGBS due to the selective damage of DNA containing unmethylated cytosines by sodium bisulfite [13] (see also Fig. 2bc).

We next plotted both EM-seq and WGBS data across all five Arabidopsis chromosomes (Fig. 4a). In general, methylation levels reported by WGBS are higher than those from EM-seq, especially in the case of $\mathrm{CHH}$ methylation, where some WGBS libraries made under suboptimal conditions (e.g. higher number of PCR cycles) suffer from severe over-estimation of methylation in the euchromatic arms of the chromosomes. Interestingly, Fig. 4a also reveals that methylation levels measured by EM-seq are higher in pericentromeres than those measured by WGBS. In the next section, we explore this further using differentially methylated region (DMR) analysis.

Arabidopsis has been shown to have two distinctive DNA methylation patterns: CG methylation in the body of protein-coding genes and all three types of methylation (CG, CHG, and $\mathrm{CHH}$ ) in repetitive DNAs such as transposable elements (TEs) $[8,9,28-30]$. In the past, metaplot analysis by WGBS has always reported some residual non-CG methylation inside gene bodies that were indistinguishable from noise $[8,9]$ due to the background non-conversion issues associated with bisulfite conversion (Fig. 1c, Additional file 1: Fig. S2). Since we now know that EM-seq has much lower background than WGBS, we ran the same metaplot analysis with EM-seq data and observed much reduced $\mathrm{CHG}$ and $\mathrm{CHH}$ methylation levels across gene bodies (Fig. 4b). The observed levels were still an order of magnitude higher than the pure background noise that can be inferred from chloroplast methylation, especially in the case of CHG $(1.16 \%$ CHG methylation on average over gene body compared 


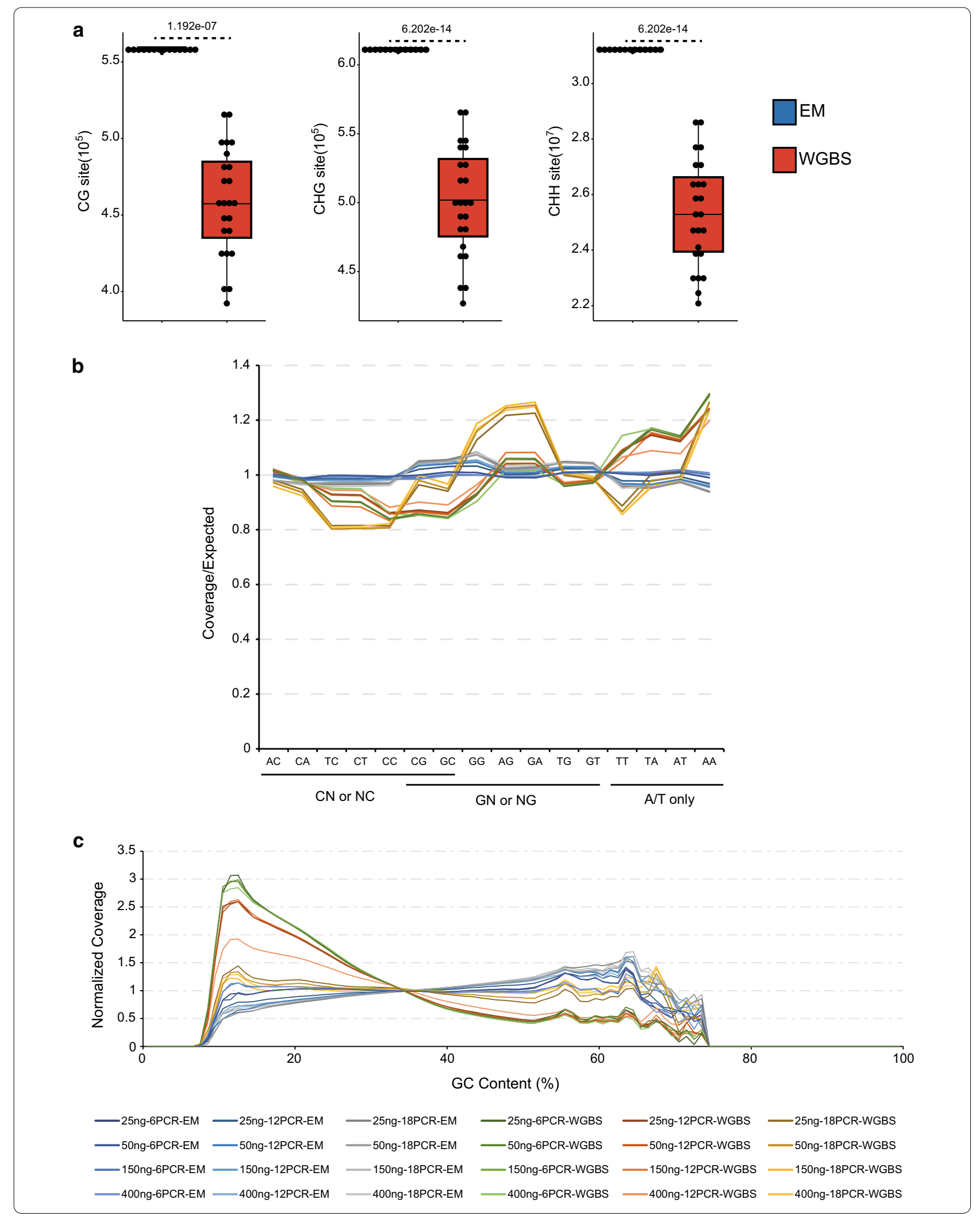




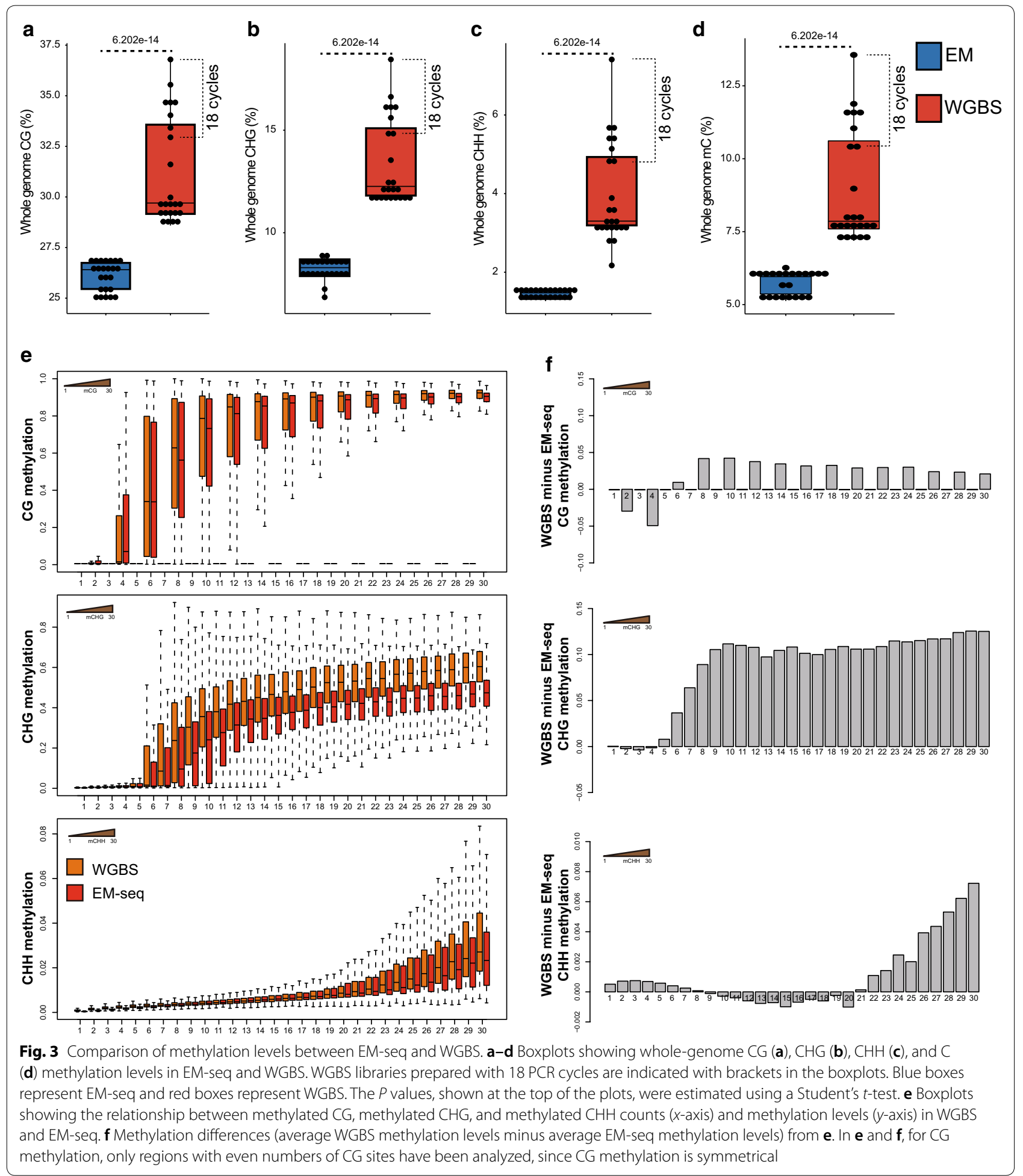

to $0.22 \%$ background) (Additional file 1: Fig. S2, Additional file 2: Table S1c), consistent with the known presence of low levels of non-CG methylation in gene bodies [31]. In terms of methylation in TEs, EM-seq produces similar metaplot profiles as WGBS, albeit with lower levels (especially for $\mathrm{CHG}$ and $\mathrm{CHH}$ (Fig. 4c), which is expected since WGBS tends to overestimate CHG and CHH methylations (Fig. 3, Additional file 3: Table S2)). 
(See figure on next page.)

Fig. 4 Comparison of methylation pattern between EM-seq and WGBS. a Chromosomal distribution of CG, CHG, and CHH methylations. Methylation levels are calculated in Arabidopsis chromosomes divided into $100 \mathrm{~Kb}$ bins. b Metaplots of CG, CHG, and CHH methylations over genes and $1 \mathrm{~Kb}$ flanking sequences. c Metaplots of CG, CHG, and CHH methylations over TEs and $1 \mathrm{~Kb}$ flanking sequences. $\mathbf{d}$ Metaplots of CG, CHG, and $\mathrm{CHH}$ methylations over PolV ChIP-seq peaks and $1 \mathrm{~Kb}$ flanking sequences. In a-d, colored lines represent different library preparations

We also compared the methylation patterns and levels in the chromosomal plots and metaplots containing only the best WGBS library (400 ng input with 12 cycles of PCR) and its corresponding EM-seq library (Additional file 1: Fig. S3). As expected, the methylation differences between this pair of libraries were smaller than the differences when other WGBS libraries are included in the comparison. Nevertheless, the basic patterns are the same as described above (see Fig. 4).

Since CHG and $\mathrm{CHH}$ methylations are maintained by RNA-directed DNA methylation (RdDM) [29], we looked at methylation in genomic regions bound by POLYMERASE V (PolV), which are often used as a proxy for RdDM target loci [32, 33]. CHG and CHH methylations over the PolV ChIP-seq peaks were elevated to various extents in different WGBS libraries, while all the EM-seq libraries show similar levels (Fig. 4d).

As an example of a gene with a well-studied methylation pattern, we looked at methylation in the FLOWERING WAGENINGEN (FWA) locus, a target of RdDM [34-36]. While methylated cytosines in non-CG contexts were detected at low levels throughout the FWA gene in almost all of the WGBS datasets, EM-seq data clearly show that non-CG methylation only exists in the promoter/beginning of coding sequence (CDS) region of FWA (Additional file 1: Fig. S4a), where the known patch of $\mathrm{RdDM}$ is known to occur. Even when using only data from the best WGBS library (400 ng input with 12 cycles of PCR), we still see trace amounts of non-CG methylation downstream of the promoter/beginning of CDS region of $F W A$; the same places show no non-CG methylation in EM-seq data (Additional file 1: Fig. S4b).

\section{Differentially methylated region analyses in EM-seq and WGBS}

We performed pairwise differential methylation region (DMR) analysis both within the various datasets of EMseq or WGBS and across EM-seq and WGBS datasets. Orders of magnitude fewer DMRs are called within the EM-seq libraries than within WGBS libraries (Additional file 1: Fig. S5ab). A larger number of DMRs arose in comparisons between the EM-seq libraries made from the least input DNA amount and the most input DNA amount and between the EM-seq libraries made with the lowest number of PCR cycles and the highest number of PCR cycles (Additional file 1: Fig. S5a). The same trends were observed in comparisons between WGBS libraries, although the WGBS comparisons produce much larger numbers, especially in the case of $\mathrm{CHG}$ and $\mathrm{CHH}$ methylation (Additional file 1: Fig. S5b).

When comparing called DMRs between EM-seq libraries and WGBS libraries made from the same amount of input DNA and with the same number of PCR cycles, we noticed that there are many more WGBS hyper-DMRs (higher methylation in WGBS libraries) than EM-seq hyper-DMRs (Fig. 5a-c). WGBS libraries with 18 cycles of PCR were clearly the outliers, since they tended to have more WGBS hyper-DMRs than other conditions, and the situation is made worse by combining 18 cycles of PCR with $400 \mathrm{ng}$ of input DNA (Fig. 5a-c). Therefore, when making WGBS libraries, excess PCR amplification should be avoided, especially if starting with plenty of DNA. There are 7.94 (4123/519) times as many WGBS hyper-CG DMRs as EM-seq hyper-CG DMRs, while the numbers for CHG and $\mathrm{CHH}$ are $405.99(110834 / 273)$ and $802.81(660713 / 823)$ times, respectively, suggesting that WGBS has more enrichment of CHG and $\mathrm{CHH}$ hyper-DMRs than CG hyper-DMRs (Fig. 5d, Additional file 4: Table S3). This fits with our previous finding that $\mathrm{CHG}$ and $\mathrm{CHH}$ methylations are more over-estimated by WGBS than is CG methylation (Figs. 3, 4). For EMseq hyper-DMRs, we saw some variation in DMR numbers among different library preparation conditions, for example, more hyper-DMRs were seen between libraries with 12 cycles of PCR (Fig. 5a-c); however, we suspect that this is rather due to the variation in WGBS libraries than the difference between WGBS and EM-seq libraries (since WGBS libraries have much higher variation among themselves than do EM-seq libraries (Additional file 1: Fig. S5ab)).

We next plotted the methylation levels in the defined EM-seq and WGBS hyper-DMRs (Fig. 5e). Interestingly, EM-seq hyper-CG and CHH DMRs on average have higher methylation levels than WGBS hyper-CG and $\mathrm{CHH}$ DMRs, respectively. The methylation level in EM-seq hyper-CHG DMRs is lower than that in WGBS hyper-CHG DMRs, which is the opposite of what is observed in EM-seq hyper-CG and CHH DMRs (Fig. 5e). There are very few EM-seq hyper-CHG DMRs, and many of them are obtained from comparison of EM-seq and WGBS in two conditions (400 ng input, 6 and 12 cycles) (Fig. 5b), which could skew the result. Furthermore, most 

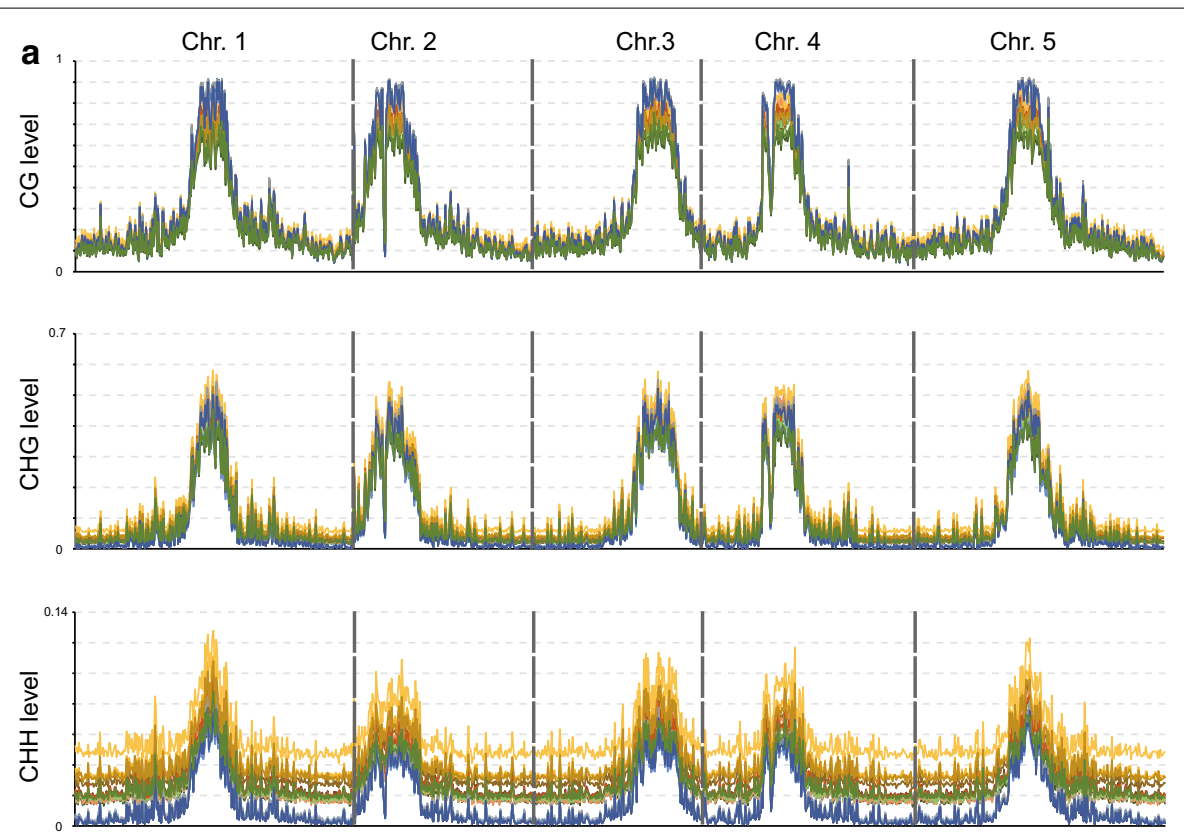

b
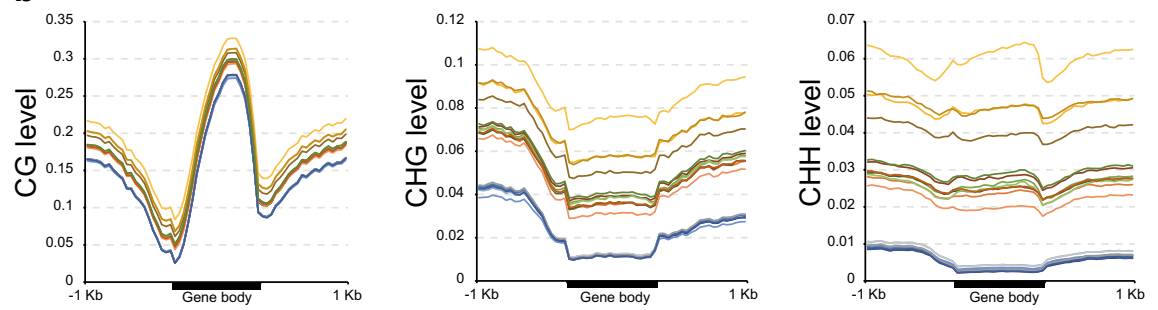

C
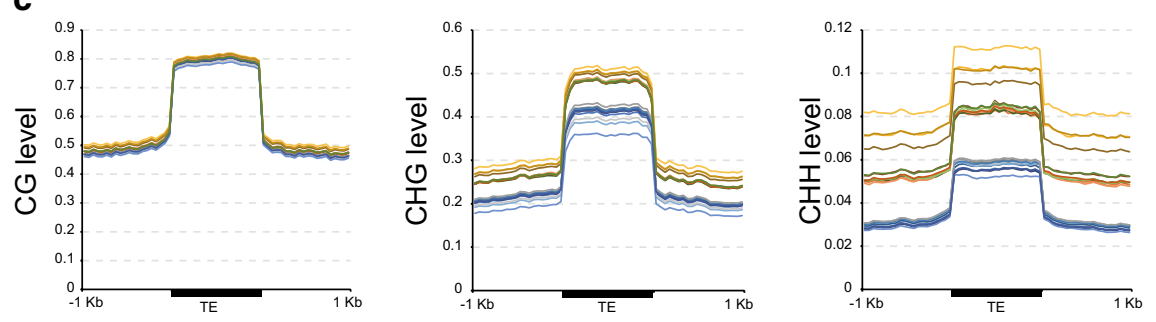

d
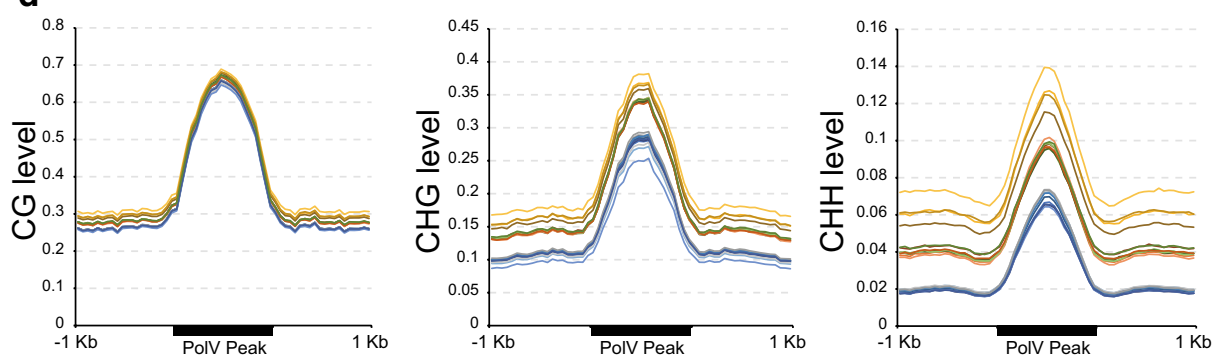

$$
\begin{aligned}
& \text {-25ng-6PCR-EM -25ng-12PCR-EM -25ng-18PCR-EM -25ng-6PCR-WGBS -25ng-12PCR-WGBS -25ng-18PCR-WGBS } \\
& \text {-50ng-6PCR-EM -50ng-12PCR-EM -50ng-18PCR-EM -50ng-6PCR-WGBS -50ng-12PCR-WGBS -50ng-18PCR-WGBS } \\
& \text {-150ng-6PCR-EM -150ng-12PCR-EM -150ng-18PCR-EM - 150ng-6PCR-WGBS -150ng-12PCR-WGBS -150ng-18PCR-WGBS } \\
& \text {-400ng-6PCR-EM —400ng-12PCR-EM -400ng-18PCR-EM -400ng-6PCR-WGBS -400ng-12PCR-WGBS -400ng-18PCR-WGBS }
\end{aligned}
$$



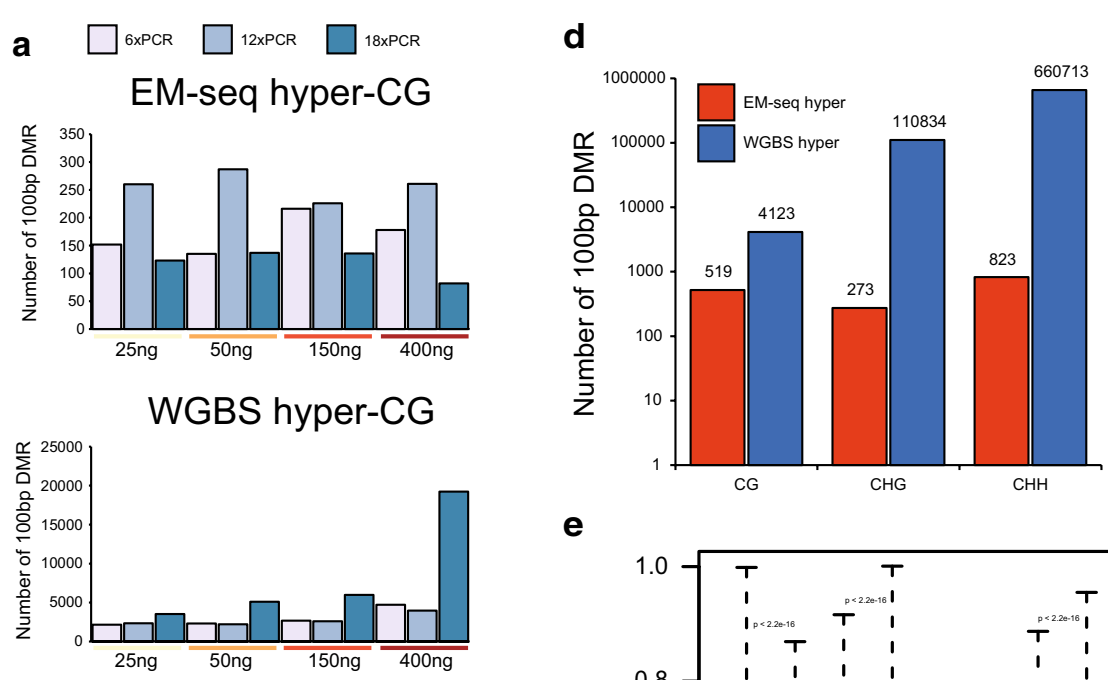

$\mathbf{e}$
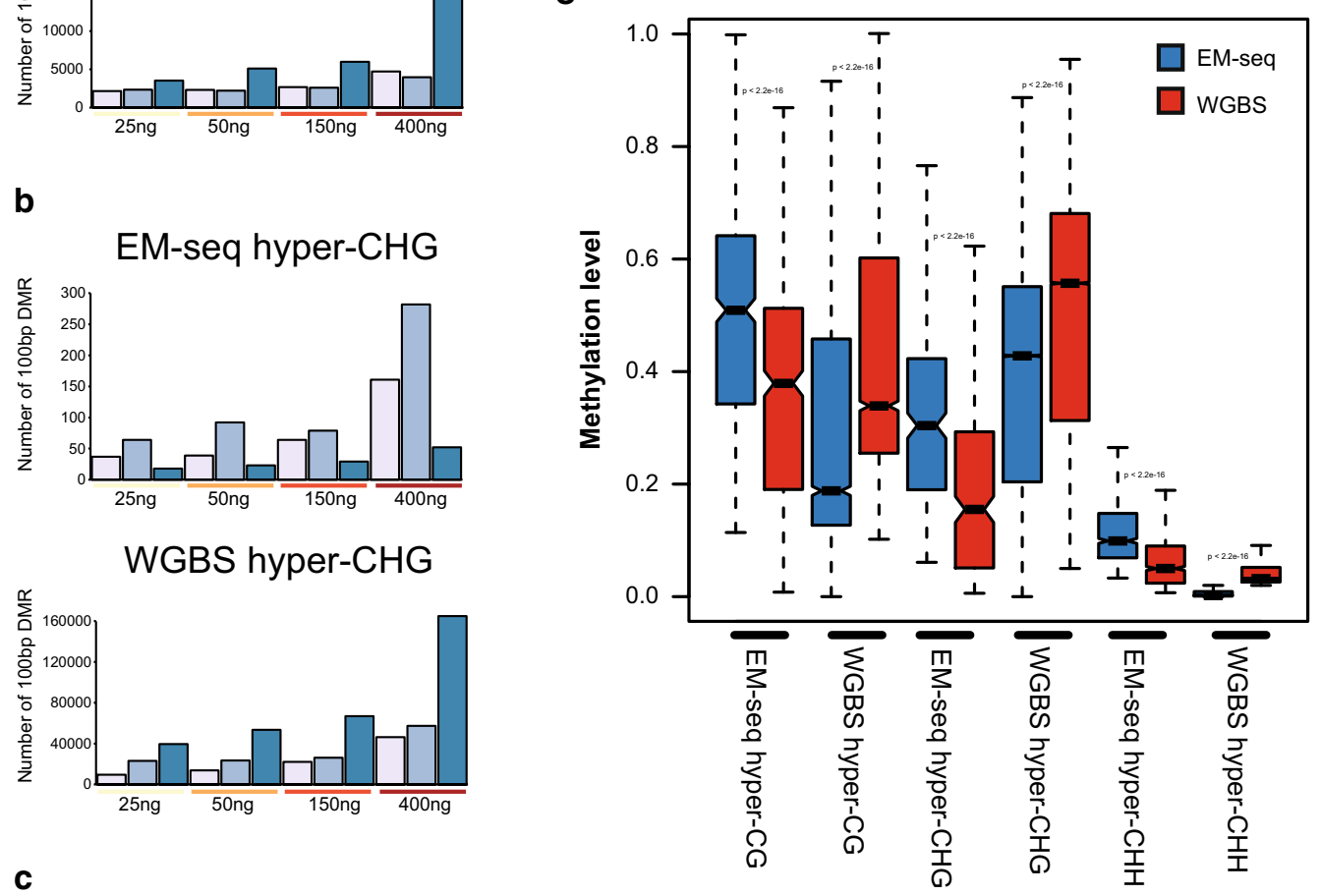

C

EM-seq hyper-CHH
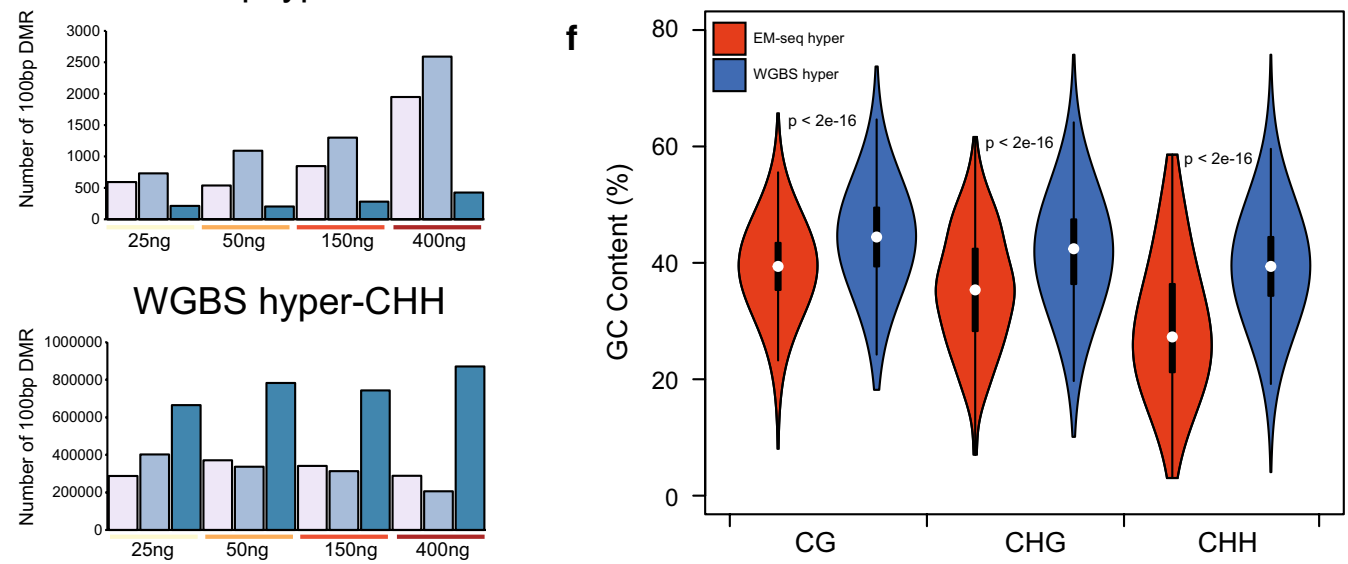

Fig. 5 Differences in differentially methylated regions (DMRs) between EM-seq and WGBS. a-c CG (a), CHG (b), and CHH (c) hyper-DMR numbers of pairwise comparison between WGBS and EM-seq from each of the different library preparation conditions. $\mathbf{d}$ Total DMR numbers of all EM-seq vs. all WGBS. e Methylation levels of EM-seq and WGBS hyper-DMRs. $\mathbf{f}$ GC contents of EM-seq and WGBS hyper-DMRs. In e and $\mathbf{f}$, the $P$ values, shown at the top of the plots, were estimated using a Student's t-test 
of the WGBS hyper-CHG DMRs are in pericentromeric heterochromatin regions (Additional file 1: Fig. S5c) with high levels of methylation (see Fig. 4a). GC content analyses in DMRs indicate that EM-seq hyper-DMRs have significantly lower GC content than WGBS hyper-DMRs (Fig. 5f). One extreme case is the mitochondria chromosome (chrM), which has a 10\% higher GC content than the five nuclear chromosomes (Additional file 1: Fig. S6a). The relative difference in methylation levels between EM-seq and WGBS in chrM is larger than that in other chromosomes (Additional file 1: Fig. S6b), and in fact the majority of the chrM is called as WBGS hyper-DMRs (Additional file 5: Table S4). The methylation levels in chrM are generally very low as determined by EM-seq (Additional file 1: Fig. S6b), which fits with the previous observation that WGBS hyper-DMRs tend to have lower methylation levels (Fig. 5e). We reasoned that in WGBS hyper-DMRs in nuclear chromosomes there are likely many sites that should have no or very low methylation (like chrM); this would make the average methylation levels in WGBS hyper-DMRs low, except for WGBS hyperCHG DMRs for above-mentioned reasons (see Fig. 5e, Additional file 1: Fig. S5c).

Since GC content also greatly affects coverage (Fig. 2c), we wondered if over- (mainly $\mathrm{CHG}$ and $\mathrm{CHH}$ ) and underestimating (mainly CG) methylation by WGBS could be linked to coverage. First, we generated heatmaps and coverage plots of all the libraries across PolV ChIP-seq peaks, because these are the places with large increases in CHG and $\mathrm{CHH}$ methylation in WGBS libraries (Fig. 4d). We found that for EM-seq libraries, although coverage fluctuates, the ranges are typically quite small. On the other hand, there is a significant increase in coverage coinciding with the center of PolV ChIP-seq peaks for all WGBS libraries (Additional file 1: Fig. S7). A reasonable explanation for this is that PolV-binding sites are targets of $\mathrm{RdDM}$ and contain methylated CHGs and CHHs that are not converted by bisulfite treatment; they, therefore, become better templates for PCR amplification (see data from previous sections) and gain higher coverage than their surrounding regions. Therefore, methylation levels and coverage are positively correlated in this case. The majority of the EM-seq hyper-CG DMRs are located within pericentromere heterochromatins and are highly methylated (Fig. 4a, Fig. 5e, Additional file 1: Fig S8a), but occasionally they can be found in euchromatin regions and within genes (Additional file 1: Fig. S8b). We chose the best WGBS library (400 ng input with 12 cycles of PCR) and its corresponding EM-seq library and analyzed their coverage across EM-seq hyper-DMRs (Additional file 1: Fig. S8c-e). Interestingly, WGBS coverage spikes in EM-seq hyper-DMRs as well, and EM-seq coverage again shows only small fluctuations. The low GC content of EM-seq DMRs (Fig. 5f) could be the cause of high coverage in WGBS libraries (see Fig. 2c). Moreover, according to Fig. 2c, in regions where GC content is low $(\sim 30 \%$ or less, approximately the range of GC content found in EM-seq hyper-DMRS, see Fig. 5f), a further reduction in GC content (e.g., that caused by bisulfite treatment) will induce a sharp increase in WGBS coverage. This effect likely outweighs the PCR preference for unconverted DNA and causes WGBS to under-estimate methylation levels in these regions.

\section{Methylation differences between Arabidopsis leaves and flowers detected by EM-seq}

We applied the EM-seq method to analyze methylation differences between Arabidopsis leaves and flowers. We used 150 ng input DNA and 6 cycles of PCR, and generated 4 replicates for each tissue. Overall, CG and CHG methylation levels were slightly higher in flowers than in leaves, and $\mathrm{CHH}$ methylation was about the same in both tissues (Fig. 6a). Metaplots in genes reveal that there are very small differences between leaves and flowers in gene body methylation levels-CG plots are almost identical and $\mathrm{CHG}$ and $\mathrm{CHH}$ are close to noise levels in both tissues (Fig. 6b). For TEs, CG is very slightly increased and $\mathrm{CHH}$ is very slightly decreased in flowers compared to leaves (Fig. 6c), and the same trends can be observed in pericentromeric heterochromatins in chromosomal methylation plots (Fig. 6d). The most striking finding from these analyses is that CHG methylation is significantly higher in flowers than in leaves (Fig. $6 \mathrm{~cd}$ ). Consistent with this, a much larger number of flower hyper-DMRs are called in the CHG context than in CG or $\mathrm{CHH}$ (Fig. 7a, Additional file 6: Table S5). Flower hyper-CHG DMRs are located mainly in pericentromeric heterochromatins and not inside or close to genes (Figs. 6d, 7bc). DMRs for non-CG methylations are not enriched within genes, as non-CG methylations are usually not found in the genes (Figs. 6b, 7c).

CHG methylation is mainly mediated by CHROMOMETHYLASE2 (CMT2) and CMT3 via a self-reinforcing loop involving histone H3K9 methylation [22, 37, 38]. Indeed, when examining the transposons in heterochromatin regions (that have flower hyper-CHG DMRs), we observed a higher level of H3K9me2 by ChIP-seq in flowers than in leaves, whereas there was very little change in the H3K9me2 levels of euchromatic TEs that did not show flower hyper-CHG DMRs (Fig. 7d). We note that the absolute level of $\mathrm{H} 3 \mathrm{~K} 9 \mathrm{me} 2$ is much higher in heterochromatin regions than in chromosome arms (Fig. 7d), which is a characteristic of the Arabidopsis epigenome [39]. These results are consistent with a more active CHG and H3K9me2 self-reinforcing loop in flowers affecting heterochromatic TEs. 


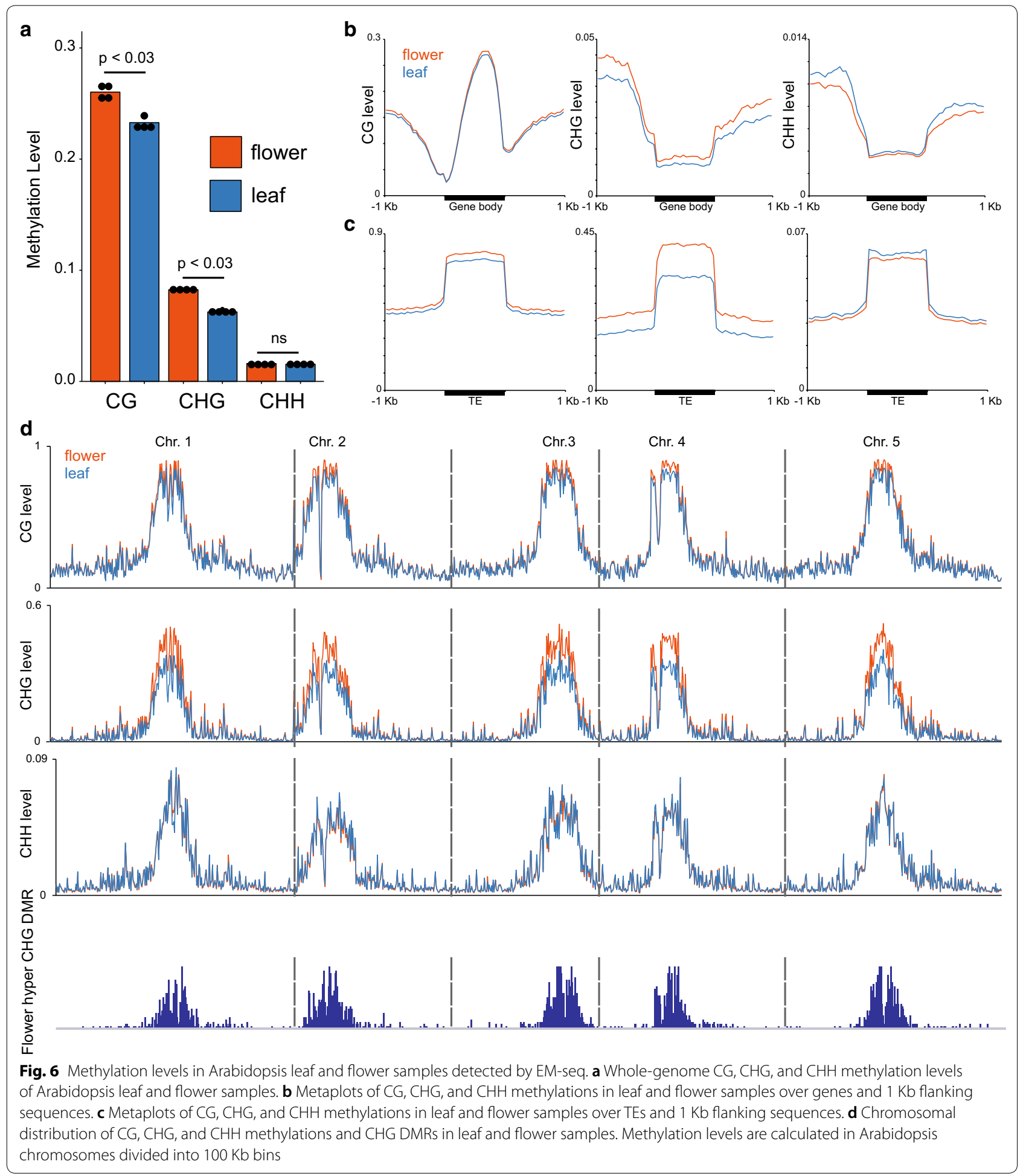

Although the overall differences in CG methylation within gene bodies are minimal between leaves and flowers (Fig. 6b), hyper-CG DMRs from both flowers and leaves were enriched in gene exons and introns (Fig. 7c; see an example in Additional file 1: Fig. S9a). Indeed, when we plotted CG methylation on genes with hyperCG DMRs in either leaves (Additional file 1: Fig. S9b, left panel) or flowers (Additional file 1: Fig. S9b, right 

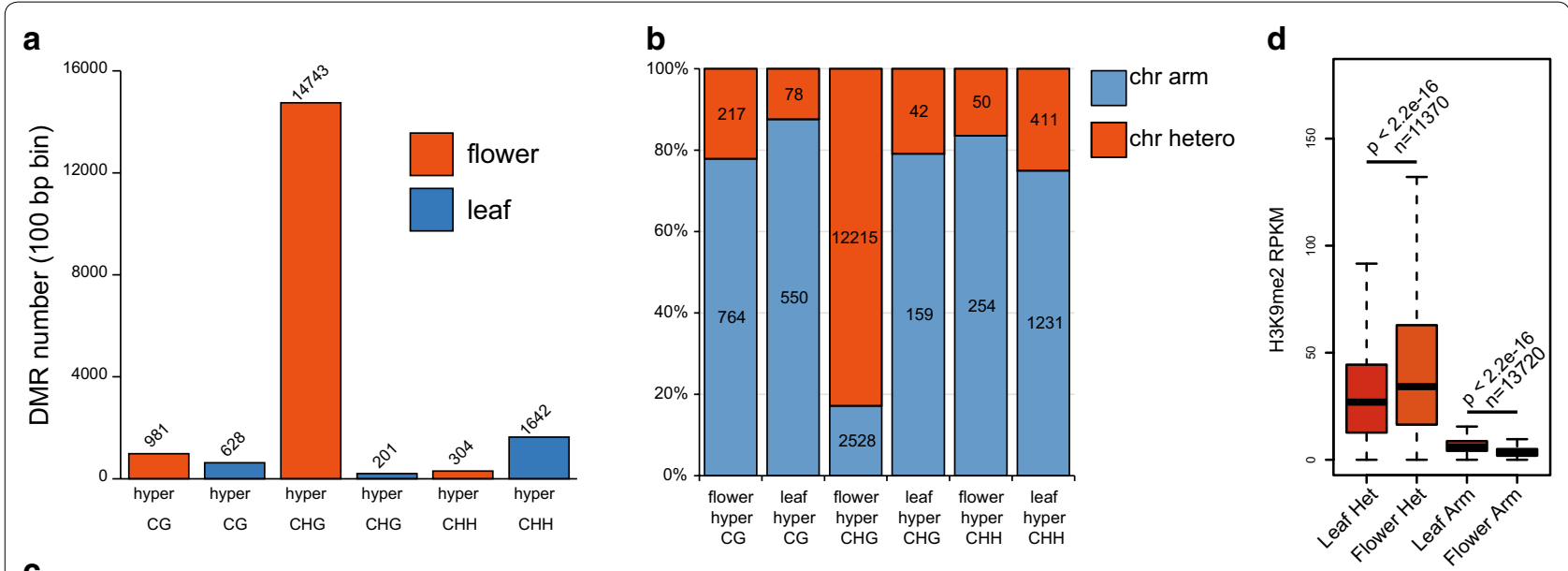

C

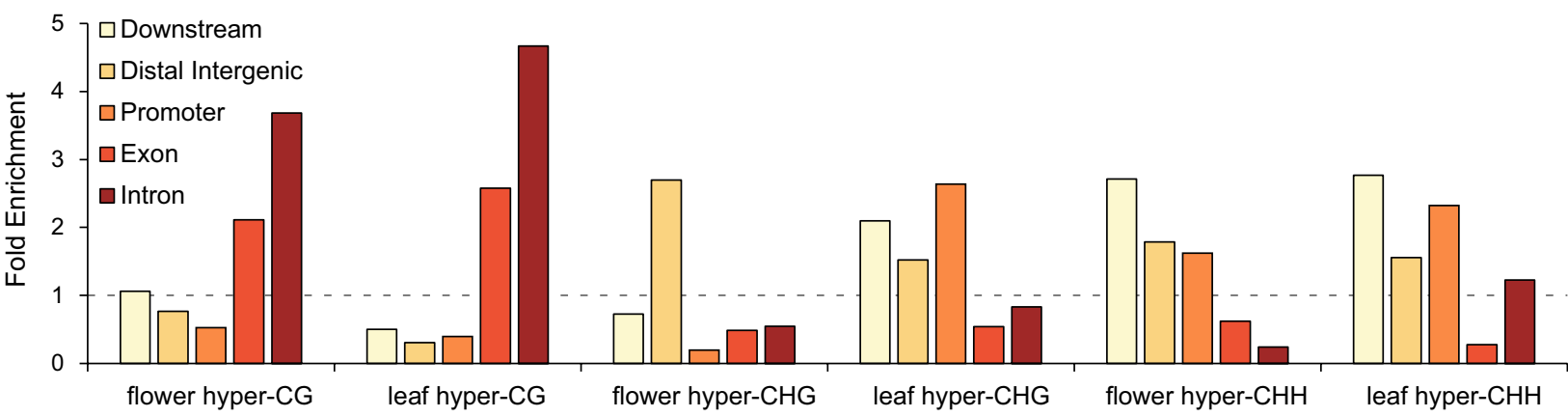

Fig. 7 DMRs and H3K9me2 levels in leaf and flower samples. a Total number of CG, CHG, and CHH hyper-DMRs in flower and leaf samples. b Distribution of CG, CHG, and CHH hyper-DMRs in chromosomal arms and heterochromatins. c Enrichment of CG, CHG, and CHH hyper-DMRs in various regions relative to genes. Expected level (1, marked by dotted line) is estimated based on genomic distribution of randomly selected regions of equal size from Arabidopsis genome. $\mathbf{d}$ H3K9me2 levels in TEs from chromosomal arms (without flower hyper-CHG DMRs) and heterochromatins (with flower hyper-CHG DMRs)

panel), we observed clear differences. Previous studies have shown that gene body-methylated genes in plants are often house-keeping genes, constitutively expressed, and exhibit moderately high expression levels [28, 40, 41]. We found that majority of the genes with hyper-CG methylations in either leaves or flowers are differentially expressed between leaves and flowers (Additional file 1: Fig. S9c). However, this does not seem to be specific, as we obtained similar results when looking at randomly selected genes (data not shown). This and the fact that both upregulated and downregulated genes in both tissues can show increased body CG methylation (Additional file 1: Fig. S9c) suggests that gene body methylation does not directly regulate the expression of these genes.

\section{Discussion}

Bisulfite sequencing, despite being the gold standard for methylation detection, is known to have shortcomings, including DNA damage, false positives due to non-conversion, uneven and missing coverage, and biased representation of methylated versus unmethylated DNA in the final library. In this study, we performed detailed analyses of these aspects and compared the results from WGBS to those from EM-seq, a newly developed, enzyme-based, bisulfite-free method for methylation detection. Our WGBS findings are consistent with those in previously published literature [7, 11-13, 21, 25-27]. In all the comparisons between EM-seq and WGBS, EM-seq appears to be mostly free of the problems that WGBS has, suggesting that it is a superior method. In addition, library preparation conditions such as input DNA amount and number of PCR amplification cycles have very little effect on the results of EM-seq, while the results of WGBS are greatly affected by these parameters, even when all WGBS libraries are generated in a single batch. Therefore, we propose that EM-seq is more desirable than WGBS, especially for big data projects that require integration of datasets obtained across a wide variety of source materials, locations and time points, and processed by personnel with different levels of expertise.

Because of its high reproducibility and low background, EM-seq is also suitable for projects aimed at 
revealing subtle methylation changes in different samples and/or conditions. We used EM-seq to study methylation differences between Arabidopsis leaves and flowers, and showed that DMRs can be called with high confidence even in places where the two tissues have very similar methylation levels. This approach can be expanded to other tissues and across organisms. EM-seq can work with much lower amounts of starting DNA than WGBS (as low as $100 \mathrm{pg}$ [20]), which makes it ideal for singlecell methylome studies. Currently, bisulfite is used in these studies $[42,43]$. Based on the performance of EMseq observed here, we expect that substituting bisulfite with TET2 and APOBEC3A will greatly enhance the success rate of single-cell methylome library generation and increase the coverage per cell.

It is worth noting that after methylated cytosines are oxidized by TET family enzymes to 5 -fCs and 5-caCs, a different approach can be taken to differentiate methylated from unmethylated cytosines. In a recently published TET-assisted pyridine borane sequencing (TAPS) procedure [44], TET1 is used in the first step (to catalyze a similar reaction to that catalyzed by TET2 in EM-seq), and pyridine borane is used in the second step to convert 5-fCs and 5-caCs to dihydrouracils (DHU). DHUs basepair with adenines during PCR and, thus, are amplified as thymines. A set of new bioinformatics tools is needed for TAPS, since in TAPS data methylated cytosines appear as thymines and unmethylated cytosines stay as cytosines, which is the opposite to WGBS and EM-seq. TAPS shows promise in overcoming many of the issues of WGBS; however, since it introduces a different skewed base composition landscape for the current library preparation reagents, sequencers, and bioinformatics tools to deal with, it could potentially lead to complications.

There are new developments in long-read sequencing technologies that enable direct sequencing of the original DNA without fragmentation or amplification, thus bypassing the need for bisulfite treatment. 5-methylcytosines can be differentiated from other bases by the virtue of distinct polymerase kinetics $[45,46]$ or unique electronic signal characteristics $[47,48]$ displayed by different bases. Nevertheless, WGBS still compares favorably in terms of accuracy, reliability, and cost effectiveness, against these technologies, at least in their current iterations $[49,50]$. An interesting alternative to improve upon these technologies is to combine bisulfite or enzymaticbased conversion with these long-read technologies. For example, bisulfite treatment has been used in combination with PacBio SMRT sequencing [51] and TAPS has been used with both SMRT and Oxford Nanopore sequencing [50]. TET2 and APOPEC3A from the EMseq protocol can likely be adapted to the same longread technologies, and because of their non-destructive nature, they are expected to better preserve the intactness of high-molecular weight genomic DNA. However, in both of the previously published methods, because sequencing of PCR products containing newly formed thymines (which correspond to unmethylated cytosines following bisulfite treatment or methylated cytosines following TAPS treatment) outperforms sequencing of intermediates (like oxidized methylcytosines, uracils, or DHUs) in terms of sensitivity, accuracy, and minimal coverage required, the converted DNAs have to be amplified with region-specific primers (aiming to obtain products up to 10 kilobases long) before being subjected to SMRT or Nanopore sequencing. This makes it impractical to use these methods for whole-genome methylation measurement of most eukaryotic organisms. Further advancements in long-read sequencing technologies to allow unequivocal identification of the products of bisulfite, TETs, pyridine borane, or APOBECs are needed for true high efficiency, amplification-free global detection of methylation.

\section{Conclusion}

Enzymatic methyl-seq (EM-seq) uses non-destructive enzymatic reactions, utilizing TET2 and APOBEC3A to convert unmethylated (but not methylated) cytosines to uracils. This approach generates the same product as bisulfite treatment, which can then be sequenced and analyzed in the same way. Here, we showed that compared to whole-genome bisulfite sequencing (WBGS), EM-seq has a higher mapping rate, lower duplication rate, and lower false-positive rate. EM-seq not only displays higher coverage than WGBS, but also the coverage is less affected by GC content. In terms of methylation detection, EM-seq covers more cytosines than WGBS and does not over-estimate methylation levels as WGBS does, especially in the context of CHG and CHH. EM-seq exhibits better consistency within libraries made from the same materials in all quality aspects examined and in report of methylation levels. Thus, in many respects, EMseq is superior to WGBS.

\section{Methods}

\section{Plant materials}

Arabidopsis plants of the Columbia-0 (Col-0) ecotype were used in this study. All plants were grown at $22{ }^{\circ} \mathrm{C}$ in a long day (16-h light, 8-h dark) growth room. Flowers and leaves were collected from 4- to 5-week-old plants.

\section{Genomic DNA extraction and fragmentation}

Genomic DNA from Arabidopsis flowers and leaves was extracted using a DNeasy Plant Mini Kit (Qiagen). Concentration of the DNA was measured by Qubit dsDNA Broad-Range Assay kit (ThermoFisher). $50 \mu \mathrm{l}$ aliquots 
containing 25, 50, 150, and 400 ng DNA were sheared by an S2 Focused-ultrasonicator (Covaris) to $\sim 200 \mathrm{bp}$ in average size using these parameters: intensity 5 , duty cycle $10 \%$, cycles per burst 200 , treatment time $120 \mathrm{~s}$.

\section{EM-seq library preparation}

EM-seq libraries were prepared from sheared DNA using an enzymatic methyl-seq kit following the manufacturer instructions (New England BioLabs). For each input amount, three PCR conditions were used: 6, 12, and 18 cycles.

\section{Whole-genome bisulfite library (WGBS) preparation}

Sheared DNA was end-repaired and ligated with TruSeq DNA single adapters (Illumina) using a Kapa DNA HyperPrep kit (Roche). Adapter-ligated DNA was converted with an EpiTect Bisulfite Kit (Qiagen). Converted DNA was PCR-amplified by MyTaq polymerase (Bioline) for 6,12 , or 18 cycles.

\section{Processing and sequencing of EM-seq and WGBS libraries}

The libraries were run on D1000 ScreenTape (Agilent) to determine the quality and size. The libraries were then purified by AMPure XP beads (Beckman Coulter) and concentrations were measured with a Qubit dsDNA Broad-Range Assay kit (ThermoFisher). Finally, libraries were sequenced on a NovaSeq 6000 sequencer (Illumina) to obtain single-end 100 bp reads.

\section{Single-nucleotide resolution methylome mapping}

WGBS and EM-seq reads were trimmed with trim galore (v0.4.2) (https://www.bioinformatics.babraham. ac.uk/projects/trim_galore/). Adapter trimmed reads were mapped to TAIR10 reference genome by BSMAP (v2.90) allowing 2 mismatches and 1 best hit (-v $2-\mathrm{w} 1)$ [52]. Reads with three or more consecutive methylated $\mathrm{CHH}$ sites were considered as unconverted reads and subsequently removed in the following analysis. Mapping and duplication rates were obtained from log files of the BSMAP pipeline.

\section{Single-nucleotide resolution methylome level calculation} DNA methylation level at each site or region was calculated by number of methylated $C$ vs. total $C$ and $T$ account. False-positive methylation levels were estimated by calculating methylation level in the Arabidopsis chloroplast genome since it is virtually unmethylated. To calculate the methylation level of genes and transposable elements, gene body or transposable element regions were divided into 20 proportionally sized bins and up/ down-stream $1 \mathrm{~kb}$ regions into $50 \mathrm{bp}$ bins. Genome-wide average methylation level of genes and transposon elements was then calculated at these bins.
Single-nucleotide resolution methylome DMR calling and annotation

Differentially Methylated Regions (DMRs) were called by methdiff.py in BSMAP with $P<0.01$ where differences in $\mathrm{CG}, \mathrm{CHG}$, and $\mathrm{CHH}$ methylation were at least 0.1, 0.05, and 0.02 (WGBS and EM-seq comparison) or 0.4, 0.2, and 0.1 (flower and leaf EM-seq comparison), respectively. Genomic distributions of DMRs were annotated by ChIPseeker [53]. Control datasets were obtained by randomly selected, equal length regions in TAIR10 genome with the bedtools shuffle function (v2.27.1). Gene expression profile was downloaded from the TAIR database (https://www.arabidopsis.org/) and visualized with $R$ package pheatmap [54]. Chromosome arm and heterochromatin regions were defined with H3K9me2 ChIP-seq data [37]. Regions highly enriched with H3K9me2 ChIPseq signal were defined as heterochromatin regions.

\section{Reads coverage analysis}

Reads coverage depth were estimated through converting mapping depth with deeptools2 (v2.5.1) [55] and plotted over PolV ChIP-seq peaks [33] or DMRs. Dinucleotide coverage was calculated with bam2nuc module integrated in Bismark (v0.18.2) [56]. Normalized read coverage related to GC content was estimated by CollectGcBiasMetrics in Picard (v2.13.2) (http://broadinstitute. github.io/picard/). $\mathrm{mC}$ density was calculated by dividing the genome into $400 \mathrm{bp}$ bins and counting methylated Cs in each bin in the CG, CHG, or $\mathrm{CHH}$ context.

\section{Chromatin immunoprecipitation (ChIP) assays}

H3K9me2 ChIP-seq in Arabidopsis leaves was previously published [57]. For H3K9me2 ChIP-seq in Arabidopsis flowers, 3 grams of Arabidopsis Col-0 wild-type unopened flower buds were collected. The nuclei were isolated from these materials for in vitro cross-linking with $1 \%$ formaldehyde. Nuclei were lysed and the chromatin was sheared with Bioruptor Plus (Diagenode). The sheared chromatins were equally separated for two ChIPs. $5 \mu \mathrm{l}$ of anti-H3K9me2 (ab1220, abcam) and antiH3 (ab1791, abcam) antibodies were added for chromatin immunoprecipitation, respectively. This experiment was performed by closely following the protocol described in a previous paper [58]. ChIP-seq libraries were prepared from DNA extracted from the ChIP experiment using Ovation Ultra Low System V2 Kit following manufacturer instructions (NuGEN). The libraries were sequenced on a HiSeq 4000 sequencer (Illumina) to obtain single-end $50 \mathrm{bp}$ reads. To assess differences in H3K9me2 level at heterochromatin and euchromatin regions, we selected heterochromatin TEs that overlap with flower hyper-CHG DMRs and euchromatic TEs that do not overlap with flower hyper-CHG DMRs and 
compared their $\mathrm{H} 3 \mathrm{~K} 9 \mathrm{me} 2$ level in leaf and flower tissue, respectively. H3K9me2 levels were calculated by converting ChIP seq reads count to RPKM with bamCoverage function in bedtools [59].

\section{Supplementary information}

Supplementary information accompanies this paper at https://doi. org/10.1186/s13072-020-00361-9.

Additional file 1: Figure S1. Schematic diagram of experimental design. Figure S2 CG, CHG, and $\mathrm{CHH}$ methylation levels detected in chloroplast genome by EM-seq and WGBS. Blue box represents EM-seq and red box represents WGBS. The P values, shown at the top of the plots, were estimated with a Student's $t$ test. Figure S3 Comparison of methylation pattern between EM-seq and WGBS libraries prepared with 400 ng DNA input and 12 cycles of PCR. a Chromosomal distribution of CG, CHG, and $\mathrm{CHH}$ methylations. Methylation levels were calculated with Arabidopsis chromosomes divided into $100 \mathrm{~Kb}$ bins. b Metaplots of CG, CHG, and $\mathrm{CHH}$ methylations over genes and $1 \mathrm{~Kb}$ flanking sequences. c Metaplots of $\mathrm{CG}, \mathrm{CHG}$, and $\mathrm{CHH}$ methylation over TEs and $1 \mathrm{~Kb}$ flanking sequences. Figure S4 Genome browser screenshots of FWA locus. a Comparison of methylation detected by EM-Seq and WGBS in libraries prepared with 50 ng DNA input. b Comparison of methylation detected by EM-Seq and WGBS in libraries prepared with 400 ng DNA input and 12 cycles of PCR. Figure S5 DMR numbers and chromosomal distribution. a DMR numbers between EM-seq libraries prepared with different conditions. b DMR numbers between WGBS libraries prepared with different conditions. c Chromosomal distribution of CHG methylation in EM-seq and WBGS libraries prepared with 400 ng DNA input and 12 cycles of PCR and WGBS hyper-CHG DMRs. Figure S6 Methylation in mitochondrial DNA. a GC contents of Arabidopsis chromosomes. $\mathbf{b} \mathrm{CG}, \mathrm{CHG}$, and $\mathrm{CHH}$ methylation levels in mitochondrial DNA. Figure S7 Heatmaps showing read coverage of EM-seq (left six columns both rows) and WGBS (right six columns both rows) over PolV ChIP-seq peaks.Figure S8 EM-seq hyper-DMRs. a,b Genome browser screenshots of EM-seq hyper-DMRs in pericentromeric TE (a) and in gene (b). c-e Heatmaps showing read coverage of EM-seq (left panels) and WGBS (right panels) over EM-seq hyper-CG (c), CHG (d), and $\mathrm{CHH}(\mathbf{e})$ DMRs. Figure $\mathbf{5 9}$ Gene body methylated genes. a Genome browser screenshots of flower hyper-CG DMRs in a gene body methylated gene. $\mathbf{b}$ Metaplots of $\mathrm{CG}, \mathrm{CHG}$, and $\mathrm{CHH}$ methylations in leaf and flower samples over genes containing hyper-CG DMRs and $1 \mathrm{~Kb}$ flanking sequences. c Clustering of expression patterns of gene body methylated genes containing hyper-CG DMRs.

Additional file 2: Table S1. Comparison of sequencing results from EM-seq and WGBS libraries. a Mapping rates and duplication rates. b Non-conversion filtering rates and coverages. $\mathbf{c}$ Methylation levels in chloroplast DNA.

Additional file 3: Table S2. Whole-genome methylation levels in EM-seq and WGBS.

Additional file 4: Table S3. DMRs between EM-seq and WGBS

Additional file 5: Table S4. DMRs in mitochondrial DNA.

Additional file 6: Table S5. DMRs between Arabidopsis leaf and flower samples.

\section{Acknowledgements}

We thank Mahnaz Akhavan for technical assistance. High-throughput sequencing was performed at the UCLA Broad Stem Cell Research Center BioSequencing Core Facility.

\section{Authors' contributions}

SF, ZZ, and SEJ conceived and designed the study. SF performed all the methylation experiments. MW performed the ChIP assays. ZZ performed all the data analyses. SF, ZZ, and SEJ wrote the manuscript. All authors read and approved the final manuscript.

\section{Funding}

This research was supported by grants from the Bill \& Melinda Gates Foundation (OPP1194889) and NIH (R35 GM130272). S.E.J. is an Investigator of the Howard Hughes Medical Institute.

\section{Availability of data and materials}

High-throughput sequencing data generated in this study can be accessed through Gene Expression Omnibus (GEO) database under accession number GSE151616.

\section{Ethics approval and consent to participate}

Not applicable.

\section{Consent for publication}

All authors consent to publication.

\section{Competing interests}

The authors declare that they have no competing interests.

\section{Author details}

${ }^{1}$ Department of Molecular, Cell and Developmental Biology, University of California at Los Angeles, Los Angeles, CA 90095, USA. ${ }^{2}$ Eli and Edythe Broad Center of Regenerative Medicine and Stem Cell Research, University of California at Los Angeles, Los Angeles, CA 90095, USA. ${ }^{3}$ Howard Hughes Medical Institute, University of California at Los Angeles, Los Angeles, CA 90095, USA.

Received: 9 July 2020 Accepted: 18 September 2020

Published online: 07 October 2020

\section{References}

1. Edwards JR, Yarychkivska O, Boulard M, Bestor TH. DNA methylation and DNA methyltransferases. Epigenetics Chromatin. 2017;10:23.

2. Greenberg MVC, Bourc'his D. The diverse roles of DNA methylation in mammalian development and disease. Nat Rev Mol Cell Biol. 2019;20(10):590-607.

3. Law JA, Jacobsen SE. Establishing, maintaining and modifying DNA methylation patterns in plants and animals. Nat Rev Genet. 2010;11(3):204-20.

4. Schubeler D. Function and information content of DNA methylation. Nature. 2015;517(7534):321-6.

5. Smith ZD, Meissner A. DNA methylation: roles in mammalian development. Nat Rev Genet. 2013;14(3):204-20.

6. Chatterjee A, Rodger EJ, Morison IM, Eccles MR, Stockwell PA. Tools and strategies for analysis of genome-wide and gene-specific DNA methylation patterns. Methods Mol Biol. 2017;1537:249-77.

7. Frommer M, McDonald LE, Millar DS, Collis CM, Watt F, Grigg GW, Molloy $\mathrm{PL}$, Paul CL. A genomic sequencing protocol that yields a positive display of 5-methylcytosine residues in individual DNA strands. Proc Natl Acad Sci USA. 1992:89(5):1827-31.

8. Cokus SJ, Feng S, Zhang X, Chen Z, Merriman B, Haudenschild CD, Pradhan S, Nelson SF, Pellegrini M, Jacobsen SE. Shotgun bisulphite sequencing of the Arabidopsis genome reveals DNA methylation patterning. Nature. 2008;452(7184):215-9.

9. Lister R, O'Malley RC, Tonti-Filippini J, Gregory BD, Berry CC, Millar AH, Ecker JR. Highly integrated single-base resolution maps of the epigenome in Arabidopsis. Cell. 2008;133(3):523-36.

10. Lister R, Pelizzola M, Dowen RH, Hawkins RD, Hon G, Tonti-Filippini J, Nery JR, Lee L, Ye Z, Ngo QM, et al. Human DNA methylomes at base resolution show widespread epigenomic differences. Nature. 2009;462(7271):315-22.

11. Li Q, Hermanson PJ, Springer NM. Detection of DNA Methylation by Whole-Genome Bisulfite Sequencing. Methods Mol Biol. 2018;1676:185-96.

12. Raizis AM, Schmitt F, Jost JP. A bisulfite method of 5-methylcytosine mapping that minimizes template degradation. Anal Biochem. 1995;226(1):161-6.

13. Tanaka K, Okamoto A. Degradation of DNA by bisulfite treatment. Bioorg Med Chem Lett. 2007;17(7):1912-5. 
14. Ito S, Shen L, Dai Q, Wu SC, Collins LB, Swenberg JA, He C, Zhang Y. Tet proteins can convert 5-methylcytosine to 5-formylcytosine and 5-carboxylcytosine. Science. 2011;333(6047):1300-3.

15. He YF, Li BZ, Li Z, Liu P, Wang Y, Tang Q, Ding J, Jia Y, Chen Z, Li L, et al. Tet-mediated formation of 5-carboxylcytosine and its excision by TDG in mammalian DNA. Science. 2011;333(6047):1303-7.

16. Smith HC, Bennett RP, Kizilyer A, McDougall WM, Prohaska KM. Functions and regulation of the APOBEC family of proteins. Semin Cell Dev Biol. 2012:23(3):258-68.

17. Suspene R, Aynaud MM, Vartanian JP, Wain-Hobson S. Efficient deamination of 5-methylcytidine and 5-substituted cytidine residues in DNA by human APOBEC3A cytidine deaminase. PLoS ONE. 2013;8(6):e63461.

18. Wijesinghe $P$, Bhagwat AS. Efficient deamination of 5-methylcytosines in DNA by human APOBEC3A, but not by AID or APOBEC3G. Nucleic Acids Res. 2012;40(18):9206-17.

19. Schutsky EK, Nabel CS, Davis AKF, DeNizio JE, Kohli RM. APOBEC3A efficiently deaminates methylated, but not TET-oxidized, cytosine bases in DNA. Nucleic Acids Res. 2017;45(13):7655-65.

20. NEB: EM-seq: Detection of DNA methylation at single base resolution from picograms of DNA. bioRxiv 2019.

21. Warnecke PM, Stirzaker C, Song J, Grunau C, Melki JR, Clark SJ. Identification and resolution of artifacts in bisulfite sequencing. Methods. 2002;27(2):101-7.

22. Stroud H, DoT, Du J, Zhong X, Feng S, Johnson L, Patel DJ, Jacobsen SE. Non-CG methylation patterns shape the epigenetic landscape in Arabidopsis. Nat Struct Mol Biol. 2014;21(1):64-72.

23. Stroud H, Greenberg MV, Feng S, Bernatavichute YV, Jacobsen SE. Comprehensive analysis of silencing mutants reveals complex regulation of the Arabidopsis methylome. Cell. 2013;152(1-2):352-64.

24. NEB: https://www.neb.com/-/media/nebus/files/application-notes/nebne xt em-seq technicalnote.pdf?rev=39e25399c23e489db144735c0f5dd515. EM-seq TechNote.

25. Chevet $\mathrm{E}$, Lemaitre $\mathrm{G}$, Katinka MD. Low concentrations of tetramethylammonium chloride increase yield and specificity of PCR. Nucleic Acids Res. 1995;23(16):3343-4

26. Oyola SO, Otto TD, Gu Y, Maslen G, Manske M, Campino S, Turner DJ, Macinnis B, Kwiatkowski DP, Swerdlow HP, et al. Optimizing Illumina next-generation sequencing library preparation for extremely AT-biased genomes. BMC Genomics. 2012;13:1.

27. Olova N, Krueger F, Andrews S, Oxley D, Berrens RV, Branco MR, Reik W. Comparison of whole-genome bisulfite sequencing library preparation strategies identifies sources of biases affecting DNA methylation data. Genome Biol. 2018;19(1):33.

28. Bewick AJ, Schmitz RJ. Gene body DNA methylation in plants. Curr Opin Plant Biol. 2017;36:103-10.

29. Matzke MA, Kanno T, Matzke AJ. RNA-directed DNA methylation: the evolution of a complex epigenetic pathway in flowering plants. Annu Rev Plant Biol. 2015;66:243-67.

30. Tran RK, Henikoff JG, Zilberman D, Ditt RF, Jacobsen SE, Henikoff S. DNA methylation profiling identifies CG methylation clusters in Arabidopsis genes. Curr Biol. 2005;15(2):154-9.

31. Wendte JM, Zhang Y, Ji L, Shi X, Hazarika RR, Shahryary Y, Johannes F, Schmitz RJ. Epimutations are associated with CHROMOMETHYLASE 3-induced de novo DNA methylation. Elife. 2019;8:e47891.

32. Johnson LM, Du J, Hale CJ, Bischof S, Feng S, Chodavarapu RK, Zhong X, Marson G, Pellegrini M, Segal DJ, et al. SRA- and SET-domain-containing proteins link RNA polymerase $V$ occupancy to DNA methylation. Nature. 2014;507(7490):124-8.

33. Liu W, Duttke SH, Hetzel J, Groth M, Feng S, Gallego-Bartolome J, Zhong Z, Kuo HY, Wang Z, Zhai J, et al. RNA-directed DNA methylation involves co-transcriptional small-RNA-guided slicing of polymerase $V$ transcripts in Arabidopsis. Nat Plants. 2018;4(3):181-8

34. Chan SW, Zilberman D, Xie Z, Johansen LK, Carrington JC, Jacobsen SE. RNA silencing genes control de novo DNA methylation. Science. 2004;303(5662):1336.

35. Gallego-Bartolome J, Liu W, Kuo PH, Feng S, Ghoshal B, Gardiner J, Zhao JM, Park SY, Chory J, Jacobsen SE. Co-targeting RNA polymerases IV and $\checkmark$ promotes efficient De Novo DNA Methylation in Arabidopsis. Cell. 2019;176(5):1068-82.
36. Soppe WJ, Jacobsen SE, Alonso-Blanco C, Jackson JP, Kakutani T, Koornneef $M$, Peeters AJ. The late flowering phenotype of fwa mutants is caused by gain-of-function epigenetic alleles of a homeodomain gene. Mol Cell. 2000;6(4):791-802.

37. Du J, Zhong X, Bernatavichute YV, Stroud H, Feng S, Caro E, Vashisht AA, Terragni J, Chin HG, Tu A, et al. Dual binding of chromomethylase domains to H3K9me2-containing nucleosomes directs DNA methylation in plants. Cell. 2012;151(1):167-80.

38. Zemach A, Kim MY, Hsieh PH, Coleman-Derr D, Eshed-Williams L, Thao K Harmer SL, Zilberman D. The Arabidopsis nucleosome remodeler DDM1 allows DNA methyltransferases to access $\mathrm{H} 1$-containing heterochromatin. Cell. 2013;153(1):193-205.

39. Bernatavichute YV, Zhang X, Cokus S, Pellegrini M, Jacobsen SE. Genomewide association of histone $\mathrm{H} 3$ lysine nine methylation with CHG DNA methylation in Arabidopsis thaliana. PLoS ONE. 2008;3(9):e3156.

40. Zemach A, McDaniel IE, Silva P, Zilberman D. Genome-wide evolutionary analysis of eukaryotic DNA methylation. Science. 2010;328(5980):916-9.

41. Zhang X, Yazaki J, Sundaresan A, Cokus S, Chan SW, Chen H, Henderson IR, Shinn P, Pellegrini M, Jacobsen SE, et al. Genome-wide high-resolution mapping and functional analysis of DNA methylation in arabidopsis. Cell. 2006;126(6):1189-201.

42. Kelsey G, Stegle O, ReikW. Single-cell epigenomics: recording the past and predicting the future. Science. 2017;358(6359):69-75.

43. Wen L, Tang F. Single cell epigenome sequencing technologies. Mol Aspects Med. 2018;59:62-9.

44. Liu Y, Siejka-Zielinska P, Velikova G, Bi Y, Yuan F, Tomkova M, Bai C, Chen L, Schuster-Bockler B, Song CX. Bisulfite-free direct detection of 5-methylcytosine and 5-hydroxymethylcytosine at base resolution. Nat Biotechnol. 2019;37(4):424-9.

45. Flusberg BA, Webster DR, Lee JH, Travers KJ, Olivares EC, Clark TA, Korlach J, Turner SW. Direct detection of DNA methylation during single-molecule, real-time sequencing. Nat Methods. 2010;7(6):461-5.

46. PacificBiosciences: Detecting DNA base modifications using single molecule, real-time sequencing. Tech Rep 2015, https://www.pacb.com/wpcontent/uploads/2015/09/WP_Detecting_DNA_Base_Modifications_Using _SMRT_Sequencing.pdf.

47. Liu Q, Fang L, Yu G, Wang D, Xiao CL, Wang K. Detection of DNA base modifications by deep recurrent neural network on Oxford Nanopore sequencing data. Nat Commun. 2019;10(1):2449.

48. Simpson JT, Workman RE, Zuzarte PC, David M, Dursi L, Timp W. Detecting DNA cytosine methylation using nanopore sequencing. Nat Methods. 2017;14(4):407-10.

49. Gouil Q, Keniry A. Latest techniques to study DNA methylation. Essays Biochem. 2019;63(6):639-48.

50. Liu Y, Cheng J, Siejka-Zielinska P, Weldon C, Roberts H, Lopopolo M, Magri A, D'Arienzo V, Harris JM, McKeating JA, et al. Accurate targeted long-read DNA methylation and hydroxymethylation sequencing with TAPS. Genome Biol. 2020;21(1):54.

51. Yang Y, Sebra R, Pullman BS, Qiao W, Peter I, Desnick RJ, Geyer CR, DeCoteau JF, Scott SA. Quantitative and multiplexed DNA methylation analysis using long-read single-molecule real-time bisulfite sequencing (SMRT-BS). BMC Genomics. 2015;16:350.

52. XiY, Li W. BSMAP: whole genome bisulfite sequence MAPping program. BMC Bioinform. 2009;10:232.

53. Yu G, Wang LG, He QY. ChIPseeker: an R/Bioconductor package for ChIP peak annotation, comparison and visualization. Bioinformatics. 2015;31(14):2382-3.

54. Kolde R: Pheatmap: pretty heatmaps. R package version 2012, 1.0.12(2).

55. Ramirez F, Ryan DP, Gruning B, Bhardwaj V, Kilpert F, Richter AS, Heyne S, Dundar F, Manke T. deepTools2: a next generation web server for deepsequencing data analysis. Nucleic Acids Res. 2016;44(W1):W160-5.

56. Krueger F, Andrews SR. Bismark: a flexible aligner and methylation caller for Bisulfite-Seq applications. Bioinformatics. 2011;27(11):1571-2.

57. Li X, Harris CJ, Zhong Z, Chen W, Liu R, Jia B, Wang Z, Li S, Jacobsen SE, Du J. Mechanistic insights into plant SUVH family H3K9 methyltransferases and their binding to context-biased non-CG DNA methylation. Proc Natl Acad Sci U S A. 2018;115(37):E8793-802. 
58. Zhong X, Hale CJ, Law JA, Johnson LM, Feng S, Tu A, Jacobsen SE. DDR complex facilitates global association of RNA polymerase $V$ to promoters and evolutionarily young transposons. Nat Struct Mol Biol. 2012;19(9):870-5.

59. Quinlan AR, Hall IM. BEDTools: a flexible suite of utilities for comparing genomic features. Bioinformatics. 2010;26(6):841-2.

\section{Publisher's Note}

Springer Nature remains neutral with regard to jurisdictional claims in published maps and institutional affiliations.
Ready to submit your research? Choose BMC and benefit from:

- fast, convenient online submission

- thorough peer review by experienced researchers in your field

- rapid publication on acceptance

- support for research data, including large and complex data types

- gold Open Access which fosters wider collaboration and increased citations

- maximum visibility for your research: over $100 \mathrm{M}$ website views per year

At BMC, research is always in progress.

Learn more biomedcentral.com/submissions 